\title{
A Preliminary Study of Information Collection in a Mobile Sensor Network
}

\author{
Yueming Hu, Qing Li \\ College of Information \\ South China Agricultural University \\ \{ymhu@, liqing1004@stu.\}scau.edu.cn
}

\author{
Fangming Liu, Gabriel Y. Keung, Bo Li \\ Dept Computer Science and Engineering \\ Hong Kong University of Science and Technology \\ \{lfxad, gabriel, bli\}@cse.ust.hk
}

\begin{abstract}
Mobile sensor networks are desirable in a variety of application scenarios, in which information collection is no doubt of great importance. In this paper, we present a mobile sensor network architecture consisting of a potentially large number of mobile sensors and a single or multiple stationary sink nodes for sensing information collection. We formulate a distinct coverage measurement problem in term of sensing information collection; we study the relevant performance and examine the effect from a variety of relevant factors through extensive simulations. We demonstrate that the performance is not only affected by the sensor mobility and the transmission range between mobile sensors and sink node(s), but also by the distribution of mobile sensors and the number and locations of sink nodes. Based on the observation and analysis, we also provide some preliminary understandings and implications for improving the information collection performance.
\end{abstract}

\section{INTRODUCTION}

Wireless sensor networks (WSNs) have been widely studied in recent years and are expected to be applied in a variety of application scenarios such as battlefield surveillance and event detections, hostile environment monitoring, and animal behavior understanding. In typical sensor networks, only static sensors are used, in which the performance of such systems such as field coverage, highly depends on the initial deployment of sensors across a geographic area (called the region of interest). Given uneven sensor distributions, some regions often remain

\footnotetext{
* The research was support in part by grants from RGC under the contracts 616406 and 616207, by a grant from NSFC/RGC under the contract N_HKUST603/07, by a grant from HKUST under the contract RPC06/07.EG27, and by a grant from Nokia Research under the contract NOKIA001-BL.06/07.
}

Permission to make digital or hard copies of all or part of this work for personal or classroom use is granted without fee provided that copies are not made or distributed for profit or commercial advantage and that copies bear this notice and the full citation on the first page. To copy otherwise, to republish, to post on servers or to redistribute to lists, requires prior specific permission and/or a fee.

Qshine08 July 28-31, 2008, Hong Kong, China Copyright 2008 ICST ISBN 978-963-9799-26-4 DOI 10.4108/ICST.QSHINE2008.3821 uncovered. In addition, this can be explored by adversaries once they gain knowledge about the deployment strategy and sensing characteristics, uncovered path(s) can be found to render the static sensor networks ineffectual [1-2].

Recent advances in robotics and low power embedded systems have made mobile sensors possible [1-2], which is believed to be capable to construct mobile sensor networks [3]. In such networks, sensors are mounted by robots, animals or other moving objects, which can sense and collect relevant information. Mobile sensors can report sensing information to sink nodes within the coverage. The randomized mobility is appealing for several reasons: 1) there is no prior knowledge of the region of interest assumed [3]; 2) it would be difficult for an adversary or intruder to remain undetected $[2-3]$; 3) perhaps more challenging in an unfriendly environment, mobile sensors may be not aware of the locations of sink nodes beforehand.

Among various aspects of challenges posed by such mobile sensor networks, the field coverage (or called area coverage) by mobile sensors has been studied [1-3]; the information collection (or called sensing data gathering) from mobile sensors, however, has not received adequate attention. Specifically, how to capture the information collection of a mobile sensor network? What factors can affect the information collection performance? What effect and sensitivity from such factors? What is the implication? We believe it is important to understand the above questions in order to make a better use of mobile sensor networks for different application scenarios.

In this paper, we study the information collection performance and examine the effect from relevant factors in a random walk mobile sensor network. Our main contributions in this study are: 1) We present a mobile sensor network architecture consisting of a potentially large number of mobile sensors with random walk mobility, and a single or multiple stationary sink nodes collecting information from the mobile sensors; 2) we introduce and formulate a distinct coverage measure (in terms of distinct mobile sensors used to be collected by sink nodes over a period of time) to capture the information collection performance; 3 ) we show through extensive simulation that the information collection performance is not only affected 
by the sensor mobility and the transmission range between the mobile sensor and sink node, but also by the initial distribution of mobile sensors, as well as the number and locations of sink nodes. Further, we find that: 1) sensor mobility and the transmission range between the mobile sensor and sink node can be exploited to improve information collection performance, while they are constrained by the limited mobile speed and transmission capability of mobile sensor; 2) in order to obtain certain level of information collection performance, more sink nodes can be deployed to compensate for the limitation of sensor mobility and transmission range; 3) for grid and random initial distributions of mobile sensors, the placement of sink nodes should take into account the area boundary.

The rest of the paper is organized as follows. Section 2 reviews the relevant work. Section 3 presents basic system architecture, mobility model and coverage in term of sensing information collection. Section 4 provides simulation results and analysis. Section 5 concludes the paper with discussion on future work.

\section{RELATED WORK}

Recently, mobile sensor networks and relevant issues such as mobility strategy, field coverage, and information collection, have received increasing attention. Liu et al. [3] studied the dynamic aspects of the field coverage of a mobile sensor network that depends on the continuous movement of mobile sensors. Compared to static sensor networks, they showed that mobile sensors following a random walk can compensate for the lack of sensors and better field coverage. A more recent study on how the quality of field coverage scales with the motion velocity and strategies of mobile sensors can be found in [2]. Wang et al. [1] proposed a hybrid network of static sensors and mobile sensors with a random walk model and showed that a small set of mobile sensors can effectively address the uneven distribution of the static sensors so as to improve the field coverage quality. A comprehensive discussion on the mobility model including random walk, random waypoint, as well as Gauss-Markov can be found in [4].

The field coverage describes how well a region of interest is monitored by sensors; the coverage can have another interpretation from an information collection perspective. Lima et al. [5] introduced the node coverage to describe the sensing data gathering performance of a static sensor network with a single mobile patrol node in terms of the expected number of sensors captured within a given time frame. Shah et al. [6] utilized randomly moving "Data Mules" to help collect the sensing data. Kalpakis et al. [7] studied the problem of finding an efficient manner to collect data from all the sensors and transmit data to the base station, such that the system lifetime is maximized. There have been also studies on reliable and power- efficient data transmission and gathering [8-9], on static sensor network with mobile sinks can be found in [10-11], in which static sensors send out data when the sink is moving around.

The focus in this paper is different from all prior works in that we consider the information collection and relevant factors in a mobile sensor network composed of potentially large number of mobile sensors and stationary sink node(s). We are particularly interested in the key factors that affect the performance of information collection.

\section{SYSTEM ARCHITECTURE}

In this section, we present the basic system architecture, mobility model and coverage in term of sensing information collection.

\subsection{The System Model}

We consider a mobile sensor network consisting of a potentially large number of mobile sensors $(M)$. The mobility of sensor nodes follows a random walk model within a 2-D geographic area $A$ to sense the environment or detect events and store relevant information. There exists a single or multiple stationary sink nodes collecting information from the mobile sensors.

In order to capture the trajectory of the mobile sensor movement, the initial locations become relevant. Specifically, we assume that, at time $t_{0}$, the initial distribution $D\left(t_{0}\right)$ of mobile sensors across the area follows a certain pattern according to different application scenarios and requirements. In this study, we consider two typical initial distributions as follows:

- Grid distribution: mobile sensors are arranged using a grid-based fashion [12] across the area, and the separation between adjacent sensors is $\sqrt{|A| / M}$. The grid layout is a natural way for the cases in which it is possible and preferable to place the sensors in particular locations at the beginning.

- Random distribution: mobile sensors are randomly and independently distributed in the area. Such an initial deployment is suitable in scenarios where prior knowledge of the area is not available [3] or the area is not under control such as airdrop in an unfriendly area.

Starting from one type of the initial distributions, we assume that each mobile sensor performs the 2-D random walk movement, which is one of the most common and widely used mobility models [4]. With this mobility model, each mobile sensor travels from its current location to a new location by randomly choosing a direction $\theta \in[0,2 \pi)$ and a speed $v \in\left[v_{\min }, v_{\max }\right]$ respectively, in each discrete 


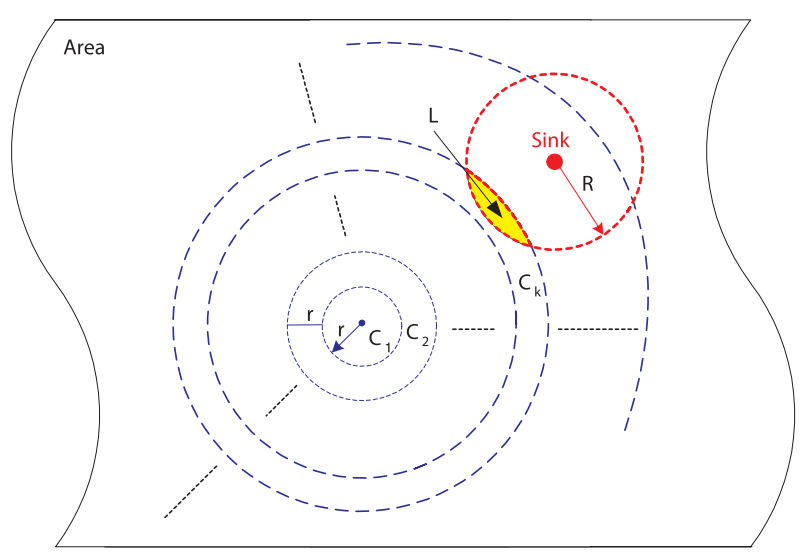

Fig. 1. A simple example for understanding the probability of a mobile sensor entering the transmission region of a sink node.

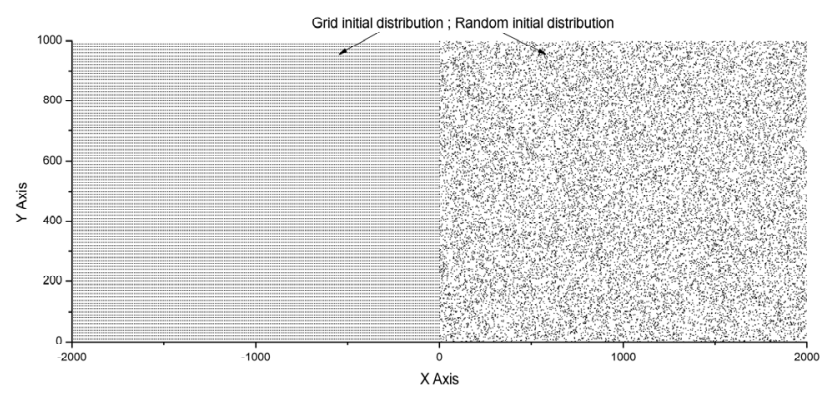

Fig. 2. Grid and random initial distribution.

time interval $\Delta t$. We further make one simplification that all mobile sensors move at a constant speed $v=v_{\max }$, so that the distance traveled in each discrete time interval can be denoted by $r=v \Delta t$. This is reasonable for the application scenario in that each mobile sensor prefers to speed up its mission progress (e.g., searching a target in a vast area), and reports its information to the sink node as soon as possible; on the other hand, as pointed out in [3], more general speed distributions can be approximated using the fixed speed scenario. Depending on different types of mobiles and application context, sensors can have different levels of speed represented by $r$. For example, sensors can be mounted on robots or animals. We show in section 5 that the information collection performance is very relevant to this factor.

Besides mobile sensors, there exist a single or multiple stationary sink nodes $\left\{S_{i}: S_{i} \in A, 1 \leq i \leq N\right\}$ for collecting information. Let the transmission range between a mobile sensor and the sink node $R$, i.e., each sink node is capable of communicating with or collecting information from those mobile sensors located within the disk of radius $R$ centered at the sink node. Hence, once a mobile sensor entering the transmission region of a sink node, we say it is covered. Although we do not explicitly consider energy and storage constrains in this paper, the limited transmission range $R$ can be viewed as energy constrain. For example, the commercially available sensors using ZigBee standards [13-14] has the data transmission capability of $30 m \sim 90 m$ in outdoor environment. We will show in section 5 that different values of $R$ can lead to different levels of information collection performance.

With limited transmission range, the sink nodes might not cover all the mobile sensors at specific time instants, but with the random walk mobility, more distinct mobile sensors can enter the transmission region of sink nodes to be collected over a period time $\left[t_{0}, t_{0}+T\right]$.

Table 1 summarizes the notations used in this paper.

Table 1. Notation

\begin{tabular}{|c|c|}
\hline Symbols & Definitions \\
\hline$A$ & $\begin{array}{c}\text { A vast 2-D geographic area called the region } \\
\text { of interest (ROI) }\end{array}$ \\
\hline$M$ & The number of mobile sensors \\
\hline$D\left(t_{0}\right)$ & $\begin{array}{c}\text { The initial distribution of mobile sensors } \\
\text { across the area } A\end{array}$ \\
\hline$\theta$ & $\begin{array}{c}\text { The moving direction of mobile sensors, } \\
\text { which is randomly chosen within }[0,2 \pi)\end{array}$ \\
\hline$v$ & $\begin{array}{c}\text { The speed of mobile sensors, and in this paper } \\
\text { we assume a constant speed }\end{array}$ \\
\hline$\Delta t$ & $\begin{array}{c}\text { The discrete time interval in which each } \\
\text { random walk movement occurs }\end{array}$ \\
\hline$r$ & $\begin{array}{c}\text { The travel distance of mobile sensors at each } \\
\Delta t, \text { which represents the speed. It depends on } \\
\text { the mobile platform of sensors and } r<<A\end{array}$ \\
\hline$N$ & The number of sink nodes, and $N \geq 1$ \\
\hline$S_{i}$ & $\begin{array}{c}\text { The position of stationary sink node } i, \text { and } \\
S_{i} \in A, 1 \leq i \leq N\end{array}$ \\
\hline$R$ & $\begin{array}{c}\text { The transmission range between the mobile } \\
\text { sensor and the sink node }\end{array}$ \\
\hline$T$ & A period of time \\
\hline
\end{tabular}

\subsection{Coverage from an Information Collection Perspective}

We now define the coverage measure from an information collection perspective as follows.

Definition 1. Coverage of distinct mobile sensors over a period of time $T$ (denoted by Coverage $(T)$ ): The total number of distinct mobile sensors that enter the transmission region of stationary sink nodes (i.e., the information of mobile sensors is collected by the stationary sink nodes and the coverage count is increased) over a period of time $\left[t_{0}, t_{0}+T\right]$.

This coverage measure reflect the information collection in the sense that given a required time period, higher coverage value implies better collection; alternatively, in 
order to collect certain amount of information, the shorter time used to meet the requirement, the higher performance we could obtain.

We next use a simple case of single sink node to illustrate the factors affecting the coverage performance. At the beginning time $t_{0}$, suppose there are $M_{0}(\geq 0)$ mobile sensors already covered by the sink node due to the initial distribution $D\left(t_{0}\right)$. According to the Euclidean distance from the position of sink node, the remaining $\left(M-M_{0}\right)$ mobile sensors, which are initially outsides the transmission region of the sink node, can be classified to subsets of $\bigcup_{i} M_{i}$, where $M_{i}$ denotes the set of mobile sensors that initially have the same Euclidean distance from the position of sink node, and

$$
\sum_{i} M_{i}=M-M_{0}
$$

For any mobile sensor initially belonging to $M_{i}$, the possible location of the sensor at time $t_{j}=t_{0}+j \Delta t$ can be characterized by the following normalized probability function:

$$
\sum_{k=1}^{j} P\left(C_{k}, t_{j}\right)=P\left(C_{1}, t_{j}\right)+P\left(C_{2}, t_{j}\right)+\cdots+P\left(C_{j}, t_{j}\right)=1 .
$$

Where those circle areas $C_{k}(k \geq 1)$ denote the area of possible locations of the sensor as time goes by, as illustrated in Fig. 1. Note that at time $t_{j}$ the farthest Euclidean distance traveled by the sensor cannot exceed $C_{j}$ under the random walk mobility model. The probability term $P\left(C_{k}, t_{j}\right)$ is the probability of "the sensor lays within $C_{k}$ at time $t_{j}$ ", and the exact probability density function is described in [15]. Thus, the probability of the mobile sensor to be covered by the transmission region of the sink node within a period of time $\left[t_{0}, t_{0}+T\right]$ can be expressed as follows:

$$
\begin{gathered}
Q\left(M_{i}, T\right) \propto \sum_{j=i}^{T / \Delta t} P\left(C_{i}, t_{j}\right) \cdot L\left(C_{i} \cap S\right)+\sum_{j=i+1}^{T / \Delta t} P\left(C_{i+1}, t_{j}\right) \cdot L\left(C_{i+1} \cap S\right)+\cdots \\
\quad+\sum_{j=T / \Delta t}^{T / \Delta t} P\left(C_{T / \Delta t}, t_{j}\right) \cdot L\left(C_{T / \Delta t} \cap S\right)
\end{gathered}
$$

Where the probability terms $L\left(C_{k} \cap S\right) \geq 0$ is related to the cross section area between the possible locations of mobile sensor and the transmission region of sink node, as shown in Fig 1.

Due to the symmetry, all the mobile sensors that initially belonging to the same $M_{i}$ would have equal probability to be covered by the transmission region of the sink node. Therefore, the expectation of coverage over a period of time $\left[t_{0}, t_{0}+T\right]$ can be expressed as follows:

$$
E[\text { Coverage }(T)]=\sum_{i} M_{i} \cdot Q\left(M_{i}, T\right) .
$$

The above simple derivation reveals that the coverage performance is related to several factors. For example, the $M_{i}$ are relevant to the number and initial distribution status of mobile sensors, as well as the position of sink node and the transmission range between mobile sensor and sink node; the possible locations of mobile sensor denoted by the $C_{k}$ and so as the probability terms $Q\left(M_{i}, T\right)$ are relevant to the random walk movement of mobile sensors and the transmission range between mobile sensor and sink node, as well as the length of the time period.

\section{SIMULATION AND ANALYSIS}

In this section, we first describe the simulation with relevant settings and then carry out a series of experiments to investigate the effect and sensitivity from various factors.

\subsection{Simulation Setting}

We develop a simulator that captures the essential aspects of the network and mobility model described in section 3. Starting from a specific initial distribution of mobile sensors, the simulator continuously calculates the coverage measure along with distinct mobile sensors entering the transmission region of sink nodes over a period of time $(T)$. Specifically, if a mobile sensor reaches the area boundary, it "bounces" off the area border according to the incoming direction [4].

Our simulator provides the flexibility of selectively controlling the configuration of various parameters including: 1) the length and width of the area $\left.\left(l^{*} w=|A|\right) ; 2\right)$ the number of mobile sensors $(M) ; 3$ ) different types of initial distribution, e.g., grid and random distributions; 4) the speed of the mobile sensor $(r)$;5) the transmission range between mobile sensor and sink node $(R) ; 6)$ the number $(N=1$ or $N>1)$ and positions of sink nodes; 7$)$ the length of the time period $(T)$.

Unless otherwise specified, we use the following default settings: for grid distribution, we define the initial separation between adjacent sensors to be 10 units resulting in 20301 mobile sensors evenly distributed in an area of size $2000 \times 1000$. For comparison, the same number of mobile sensors is used in random distribution scenario. The results are averaged over multiple runs for each corresponding set of parameter configuration.

\subsection{Single Sink}

We first consider a single sink scenario with grid and random initial distributions of mobile sensors as shown in Fig. 2. We study the information collection performance by varying two important parameters: 1) the speed of mobile sensors $(r)$ and 2 ) the transmission range between mobile sensor and sink node $(R)$.

Fig. 3 plots the percentage of mobile sensors covered against time by varying the transmission range between 


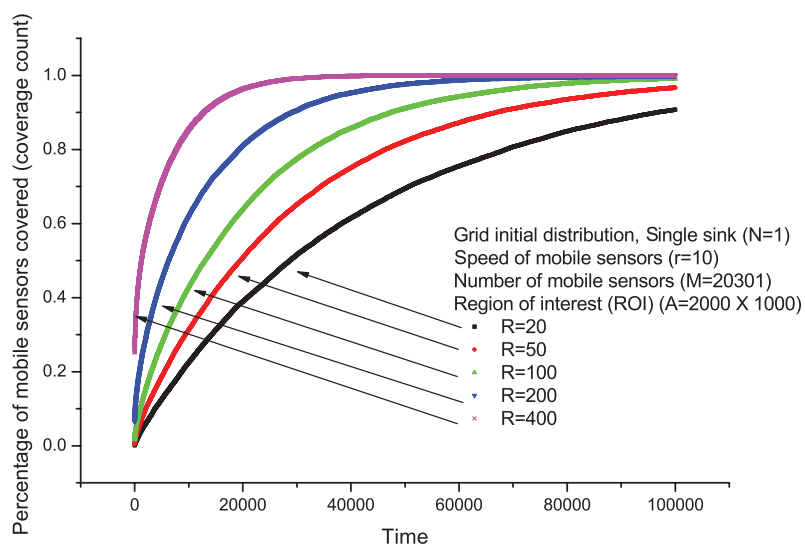

Fig. 3. Percentage of mobile sensors covered against time by varying the transmission range between mobile sensor and sink $(R)$, beginning from grid distribution.

mobile sensor and sink node $(R)$, beginning from grid initial distribution of mobile sensors. The figure shows five distinct increasing curves with different growth speed separated by transmission ranges from $R=20$ to 400 respectively ${ }^{1}$. The result demonstrates that the increase of the transmission range can sufficiently improve the information collection performance within a time period.

Fig. 4 plots the percentage of mobile sensors covered against time by varying the speed of mobile sensors $(r)$, beginning from grid initial distribution of mobile sensors. As we increase the parameter from $r=2$ to 20 respectively ${ }^{1}$, the coverage curve rises sharply with high sensitivity, which means sensor mobility can significantly affect the information collection performance. This indicates that we can exploit the speed of mobile sensors to improve the information collection performance. Compared with Fig. 3, the parameter $r$ has higher sensitivity than the parameter $R$.

Under a random initial distribution, we obtain similar conclusions that the increases of the values of $R$ and $r$ both result in increasing the coverage percentage within a fixed amount of time period, and $r$ is more sensitive than $R$.

Fig. 5 shows consistent results with that in Fig. 3, confirming that the increase of transmission range $(R)$ can improve the information collection performance, i.e., reduce the time to reach certain level of coverage percentage. Specifically, under a fixed configuration of $r$ and $R$, the time to achieve $75 \%$ coverage percentage is twice of the time to achieve $50 \%$; it would take longer time to reach higher coverage percentage. Likewise, Fig. 6 shows consistent results with that in Fig. 4, confirming that increasing the speed of mobile sensors $(r)$ can improve the information collection performance.

\footnotetext{
${ }^{1}$ We choose the ratio between $r, R$ and $|A|$ by considering their magnitudes in realistic situations [13-14].
}

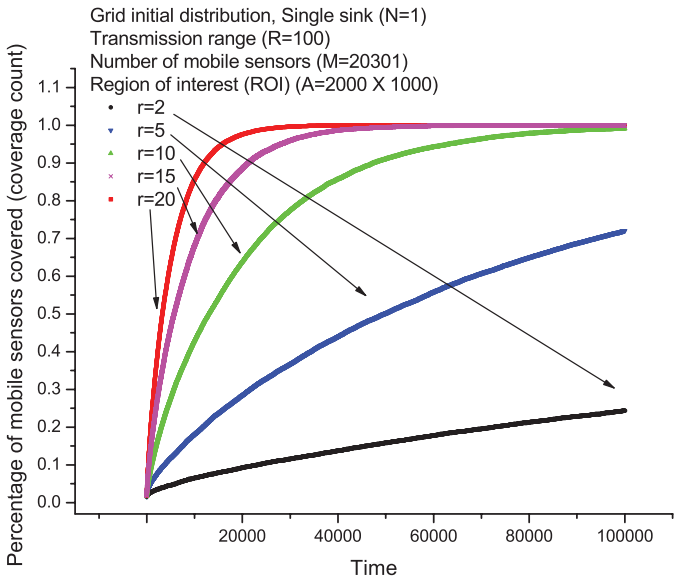

Fig. 4. Percentage of mobile sensors covered against time by varying the speed of mobile sensors $(r)$, beginning from grid distribution.

In summary, for both of the grid and random distributions, increasing the speed of mobile sensors $(r)$ and the transmission range between mobile sensor and sink node $(R)$ can help to improve the information collection performance. However, since $r$ and $R$ represent the mobile platform speed and transmission capability of mobile sensor respectively, they are both constrained in realistic applications. For example, the commercially available sensors using ZigBee standards [13-14] has a limited data transmission capability of $30 \mathrm{~m} \sim 90 \mathrm{~m}$ in outdoor environment, and the Bluetooth standards [14] has even shorter transmission range; on the other hand, the speed of mobile sensor depends on the speed limitation of different types of mobile platform, such as robots or different kinds of animals.

\subsection{Multiple Sinks}

In this subsection, we consider the multiple sink nodes scenario with grid and random initial distributions of mobile sensors. Specifically, we use the following sink nodes placement: (a) two sink nodes with line placement, i.e., at coordinates $(500,500)$ and $(1500,500)$; (b) two sink nodes with diagonal placement, i.e., at $(500,250),(1500$, $750)$; (c) four sink nodes with square placement at (500, $250),(500,750),(1500,250)$, and $(1500,750)$, respectively.

For grid initial distribution, Figs. 7 and 8 plot the percentage of mobile sensors covered against transmission range $(R)$ and the speed of mobile sensor $(r)$ respectively, at a particular time $(t=20000)$ when the percentage gap between single and multiple sink nodes clearly shown.

First, similar to single sink node scenario, the information collection performance with multiple sink nodes can be improved with the increase of $R$ and $r$.

Second, increasing the number of sink nodes $(N)$ can improve the information collection performance, which 


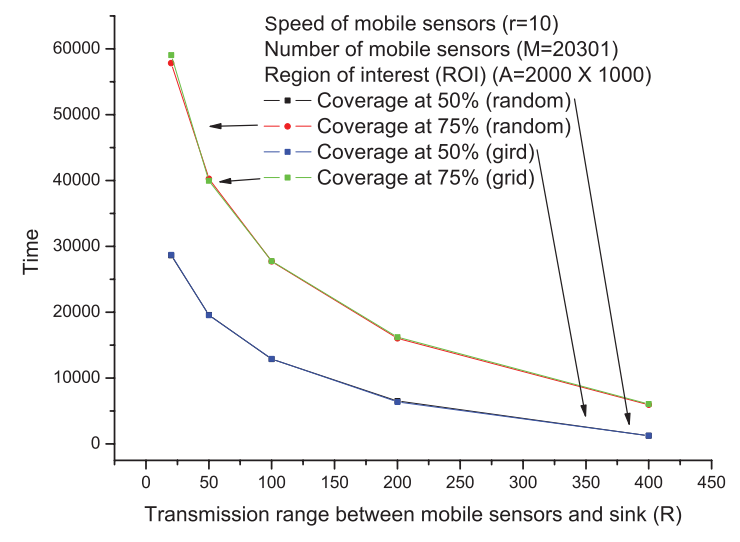

Fig. 5. Time to obtain $50 \%$ and $75 \%$ coverage against transmission range between mobile sensor and sink $(R)$, beginning from grid and random distribution.

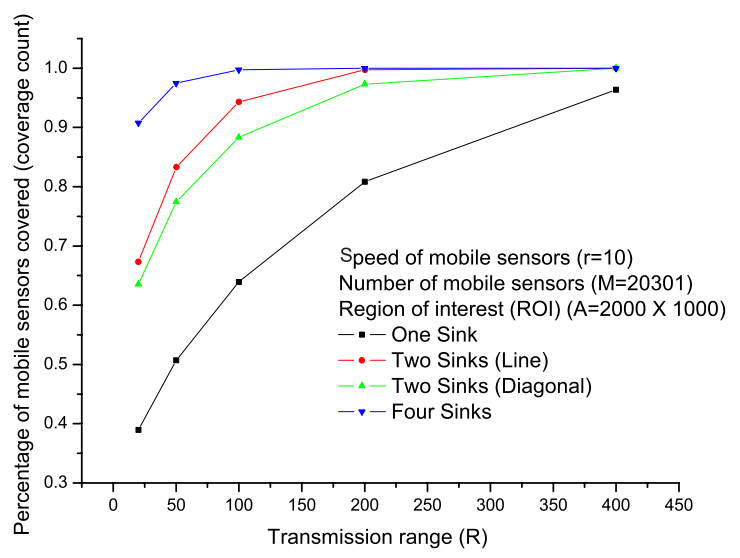

Fig. 7. Percentage of mobile sensors covered against transmission range $(R)$, at a particular time $(t=20000)$.

indicates that if $r$ or $R$ is constrained by a specific value due to physical limitation or application requirement. In order to achieve certain level of information collection performance, alternatively we can utilize more sink nodes to compensate for the limitation of $r$ or $R$.

Third, the placement of sink nodes can greatly affect the information collection performance. For example, the coverage curve of two sink nodes with line placement outperforms the two sink nodes with diagonal placement. This is because the latter is relatively closer to the area boundary; this leads to unbalanced distribution of mobile sensors around the sink and cause more sensors to travel longer distance to be covered by the sink (i.e., less probability of being covered within a given period of time under random walk mobility model).

Similar results are observed under random distribution.

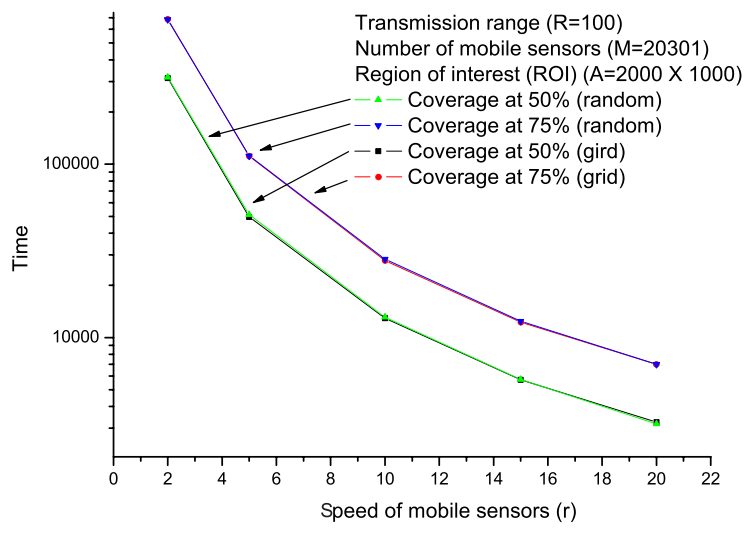

Fig. 6. Time to obtain $50 \%$ and $75 \%$ coverage against the speed of mobile sensors $(r)$, beginning from grid and random distribution.

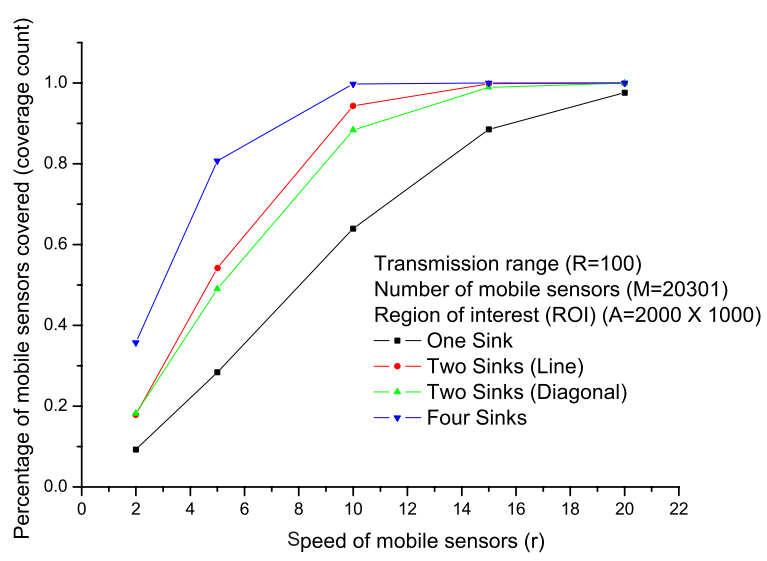

Fig. 8. Percentage of mobile sensors covered against the speed of mobile sensors $(r)$, at a particular time $(t=\mathbf{2 0 0 0 0})$.

\section{CONCLUSION AND FUTURE WORK}

In this paper, we present a mobile sensor network architecture composed of a potentially large number of mobile sensors with random walk mobility, and a single or multiple stationary sink nodes collecting information from the mobile sensors. To describe the information collection performance of such a mobile sensor network, we introduced and formulated a distinct coverage measure in term of sensing information collection. We demonstrated through extensive simulation that the information collection performance is not only affected by the sensor mobility and the transmission range between the mobile sensor and sink node, but also by the initial distribution of mobile sensors, as well as the number and locations of sink nodes.

There are several avenues for further studies: 1) to consider different mobility models such as the one [4];2) 
to study the relationship between the field coverage and the coverage in term of sensing information collection; 3) sink node placement strategy for certain sensor distribution and mobility; 4) consider other initial sensor distributions, more realistic area shapes and situations (e.g., with obstructions).

\section{REFERENCES}

[1] D. Wang, J. Liu, and Q. Zhang, "Probabilistic field coverage using a hybrid network of static and mobile sensors," in Proc. of the IWQoS'07, Chicago, IL, USA, 20-22 June, 2007.

[2] N. Bisnik, A. Abouzeid, and B. Isler, "Stochastic event capture using mobile sensors subject to a quality metric," in Proc. of the ACM MOBICOM'06, Los Angeles, September, 2006.

[3] B. Liu, P. Brass, O. Dousse, P. Nain, and D. Towsley, "Mobility improves coverage of sensor networks," in Proc. of the ACM MOBIHOC'05, Urbana Champaign, IL, May, 2005.

[4] T. Camp, J. Boleng, and V. Davies, "A Survey of Mobility Models for Ad Hoc Network Research," in Wireless Communication \& Mobile Computing (WCMC): Special Issue on Mobile Ad Hoc Networking Research, Trends and Applications, vol. 2, no. 5, pp. 483-502, September, 2002.

[5] L. Lima and J. Barros, "Random walks on sensor networks," in Proc. of the 5th International Syposium on Modeling and Optimization in Mobile, Ad hoc, and Wireless Networks (WiOpt 2007), Limassol, Cyprus, April, 2007.

[6] R. Shah, S. Roy, S. Jain, and W. Brunette, "Data mules: Modeling a three-tier architecture for sparse sensor networks," in Proc. of the IEEE Workshop on Sensor Network Protocols and Applications (SNPA), May, 2003.
[7] K. Kalpakis, K. Dasgupta, and P. Namjoshi, "Efficient algorithms for maximum lifetime data gathering and aggregation in wireless sensor networks," in Computer. Networks, vol. 42, no. 6, pp. 697-716, August, 2003.

[8] S. Lindsey and C. S. Raghavendra, "Pegasis: Power-efficient gathering in sensor information system," in Proc. of the IEEE Aerospace Conference, March, 2002.

[9] H. O. Tan and I. Korpeoglu, "Power efficient data gathering and aggregation in wireless sensor networks," in SIGMOD Rec., vol. 32, no. 4, pp. 66-71, December, 2003.

[10] A. Kansal, A. Somasundara, D. Jea, M. Srivastava, and D. Estrin, "Intelligent fluid infrastructure for embedded networks," in Proc. of the 2nd international conference on Mobile systems, applications, and services (MobiSYS), June, 2004.

[11] W. Zhao, M. Ammar, and E. Zegura, "A message ferrying approach for data delivery in sparse mobile ad hoc networks," in Proc. of the 5th ACM international symposium on Mobile ad hoc networking and computing (MobiHoc), ACM Press, pp. 187-198, May, 2004.

[12] S. Shakkottai, R. Srikant, and N. Shroff, "Unreliable sensor grids: Coverage, connectivity and diameter," in Proc. of the 22nd Annual Joint Conference of the IEEE Computer and Communications Societies (INFOCOM 03), April, 2003.

[13] http://www.rabbit.com/products/ZigBee_App_Kit/ZigBee.pdf

[14] http://en.wikipedia.org/wiki/Sensor_node

[15] W. Stadje, "The exact probability distribution of a twodimensional random walk," in Journal of Statistical Physics, vol. 46, no. 1-2, pp. 207-216, January, 1987. 